\title{
Optimization of Powerful Two-stage Screw Centrifugal Pump
}

\author{
Oleg Baturin ${ }^{1}$, Grigorii Popovv ${ }^{1}$, Daria Kolmakova ${ }^{1}$, Vasilii Zubanov ${ }^{1}$, Julia Novikova ${ }^{1}$ and Anastasia Korneeva ${ }^{1}$ \\ ${ }^{1}$ Samara University, Department of aircraft engine theory, 443086, St. 34 Moskovskoe shosse, Samara, Russia
}

\begin{abstract}
The article presents a refining method for a two-stage screw centrifugal pump by the joint usage of mathematical optimization software IOSO, meshing complex NUMECA and CFD software ANSYS CFX. The pump main parameters: high-pressure stage rotor speed was $13300 \mathrm{rpm}$; low-pressure rotor speed was $3617 \mathrm{rpm}$ by gearbox; inlet total pressure was $0.4 \mathrm{MPa}$; outlet mass flow was $132.6 \mathrm{~kg} / \mathrm{s}$ at the nominal mode. This article describes the process of simplifying the calculation model for the optimization. The parameters of camber lines of the low-pressure impeller, transition duct, and high-pressure impeller blades for two sections (hub and shroud) were chosen as optimization parameters. The blades of low-pressure impeller, transition duct and high-pressure impeller have changed during optimization. The optimization goal was the increase of the pump efficiency with preservation or slight increase in the pressure head. The efficiency was increased by $3 \%$.
\end{abstract}

\section{Introduction}

Pumps are the integral part of both industrial production and everyday human life [1]. For example, centrifugal pumps are used in water supply and water disposal systems. A special place is occupied by the screw centrifugal pumps as the devices for supplying liquid components to the rocket engine chamber. In this case, the turbo-pump unit requires both high performance and high reliability of operation. It is known [2] that more than $70 \%$ of crashes of liquid propellant rocket engine occurred due to the breakdown in the turbo-pump units.

Currently used turbo-pump units for liquid propellant rocket engines (LPRE) were designed in the 1960-1970. The geometry of the pumps was projected initially by theoretical and empirical dependencies, and they were designed by expensive experimental development of the engine/pumps.

A modern CFD programs allows the simulation of the pump workflow. After validating the simulation results, these CFD models can be used to study the effect of the pump parameters on its performance. It is also possible to optimize the pump using verified CFD model as a "black box".

An optimization of the pump geometry is carried out for the following purposes:

1) improvement of the pump performance while maintaining the pump reliability. This case can be used to boost the rocket engine. For example, it is possible to obtain a higher fuel consumption, and, consequently, a greater engine thrust at the same pump rotor rotational speed. It is possible to increase the trust-to-weight ratio of the rocket engine;
2) providing the same pump performance with the reduced pump rotor speed. In this case, the load on the rotor elements of the turbo-pump unit will be decreased;

3 ) the combination of the first two approaches.

Investigation of the working process in previous studies $[3,4]$ showed the presence of vortex zones in the high-performance pumps. The high-performance fuel pump was adopted as the study subject (Fig. 1, 2).

The fuel pump main parameters with water as the working fluid (based on experiment data) were the following:

- high-pressure stage rotor speed was $13300 \mathrm{rpm}$, low-pressure rotor speed was $3617 \mathrm{rpm}$ by gearbox;

- inlet total pressure was $0.4 \mathrm{MPa}$;

- outlet mass flow was $132.6 \mathrm{~kg} / \mathrm{s}$ at nominal mode.

Fig. 3 shows the meridional section of the fuel pump, in which vortex zones present in the following regions:

- the periphery of the inlet edge of the low-pressure screw (LPS) and high-pressure screw (HPS) (areas 1 and 4);

- the periphery of the leading edge of the lowpressure impeller (LPI) (area 2);

- the area closer to LPI stage outlet at the hub (area 3);

- the stagnation zone of the vortex near HPI hub (area 5).

Vortices in regions 1 and 4 are due to the design of the screws. At the same time, vortices 2, 3 and 5 in the pump are undesirable. Thus, the decrease of the intensity of vortices 2, 3, and 5 will increase the efficiency and pressure head of the pump. This can be achieved by changing the geometry of LPI, TP and HPI blades. 


\section{Nomenclature}

These are abbreviations of the pump parts (Fig. 1, 2):

- KID - Knee Inlet Duct;

- LPS - Low Pressure Screw passage;

- LPI - Low Pressure Impeller passage;

- TP - Transferring Passage;

- HPS - High Pressure Screw passage;

- HPI - High Pressure Impeller passage;

- VOD - Volute Outlet Duct.

Indexes:

- inlet - pump inlet;

- $i \quad$ - one of the two outlet options;

- outlet_1 - main outlet, through which the main part of the working fluid flows;

- outlet 2 - second outlet, which provides a small working fluid consumption for the rocket engine gas generator.

Other nomenclature:

- CFD - Computational Fluid Dynamics.

- $P_{-}$effective - effective power [W];

- $P_{-}$consumed - consumed power [W];

- $\mathrm{PH}_{-} \quad$ - pressure head [m];

- $\dot{m}-\quad-$ mass flow $[\mathrm{kg} / \mathrm{s}]$

- $g$ - gravity acceleration $[\mathrm{m} / \mathrm{s} 2]$;

- $\rho$ - fluid density $[\mathrm{kg} / \mathrm{m} 3]$;

- $p^{*} \quad$ - total pressure $[\mathrm{Pa}]$.

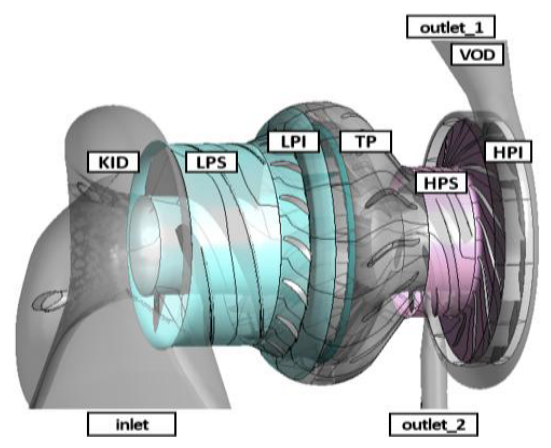

Figure 1. 3D view of the pump under investigation.

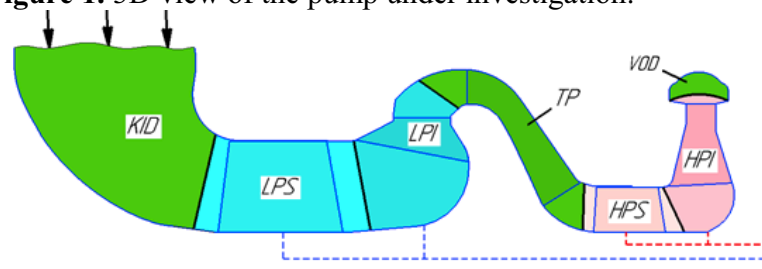

Figure 2. Meridional section of the pump under investigation (The dashed lines illustrate the mechanical links: blue lines from Hydraulic Turbine to the pre-pump, red lines - from the external turbine to the main pump).

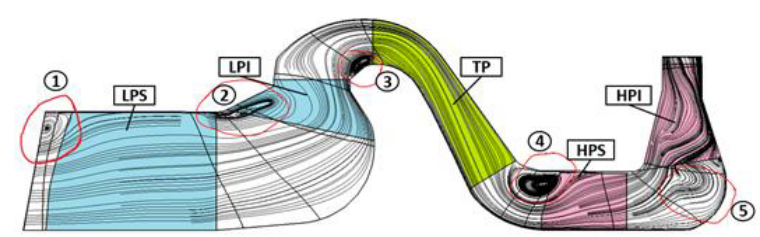

Figure 3. The meridional section of the pump with vortex zones.

\section{Methodology}

In this paper, a proven CFD model will be used from the previous study [3]. Water was used as the working fluid because the experimental test data for water were available. The proven CFD model ensures a coincidence of Pressure Head value with experimental data with the accuracy of $6.9 \%$ at nominal mode, and of Internal Efficiency $-2.0 \%$. The experimental data are presented in the form of points.

The IOSO program was used as an optimizer [5]. The optimization algorithm requires multiple iterations with CFD-model. The size of the mesh model has a direct impact on the speed of calculation. For optimization, it is important to have a mesh model that has the smallest number of elements, but at the same time adequately repeats the pressure and efficiency characteristics of the pump. This article describes the process of simplifying the calculation model for the optimization. For the final optimal variant of the pump design a calculation will be performed with the settings of the basic mesh model.

The physical processes in the pump are non-stationary [6 8]. While calculations in a stationary statement are sufficient for most engineering tasks, the parameters of efficiency and pressure head vary from iteration to iteration in high-performance pumps when calculated in a stationary calculation. It was found in previous studies that the time-averaged parameters of efficiency and pressure head for non-steady-state calculations coincide with the parameters averaged over iterations for stationary calculation.

Therefore, further calculations will be carried out in the stationary statement, with averaging the last 100 iterations by a specially created script. To calculate one task (CFD-model for optimization), 600 iterations were used, while the oscillation of parameters remained constant after 400 iterations (Fig. 4). A stock of 200 iterations was taken from considerations of a possible increase in the necessary iterations number for the task convergence. The maximum level of parameter variation for the task for the last 100 iterations was also determined by a special script. The rationale of this approach is described in the section "Comparison of the results of stationary and transient simulations of pump workflows»».

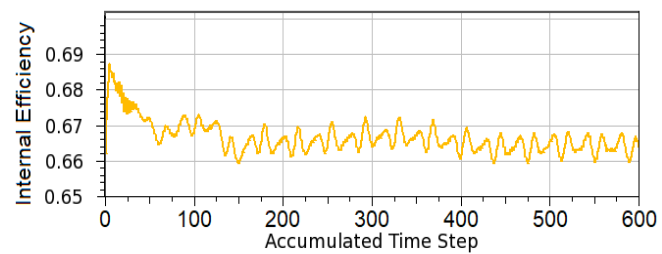

Figure 4. The internal efficiency parameter by the accumulated time step.

The volute outlet duct (VOD) of the investigated pump has two outlets: main outlet, through which the main part of the working fluid flows, and the second outlet, which provides a small working fluid consumption of $5.78 \mathrm{~kg} / \mathrm{s}$ for the rocket engine gas generator.

In general, pump internal efficiency with VOD, which has two outlets, is determined by the formula: 


$$
\text { Efficiency }=\frac{P_{\text {effective_outlet_1 }}+P_{\text {effective_outlet_2 }}}{P_{\text {romsumed }}},
$$

An effective power of $\mathrm{i}$ outlet is determined by the formula:

$$
P_{\text {effective } i}=P H_{i} \cdot \dot{m}_{i} \cdot g
$$

And Pressure Head of $\mathrm{i}$ outlet is given by formula:

$$
P H=\frac{P_{\text {outlet } i}^{*}-p_{\text {inlet }}^{*}}{\rho \cdot q}
$$

In the previous work, the definition of internal efficiency did not include effective power of the outlet_ 2 . In presented paper, this aspect was considered, and the internal efficiency values in this article is determined by the formula (1). Also, the parameters of efficiency and pressure head were calculated at the outlet of the additional nozzle for the convenient comparison of the results. In this case, the parameters will be lower than previously used, since they will include large losses due to a vortex at the outlet (Fig. 5).

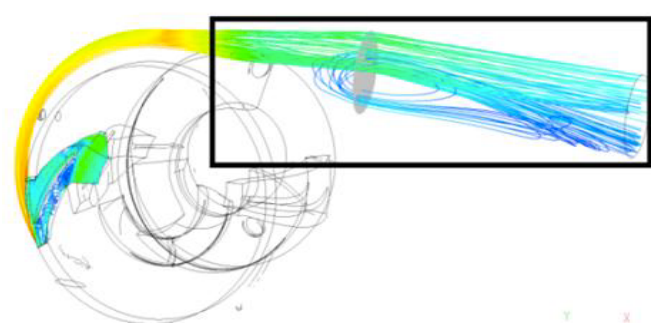

Figure 5. Vortex at the outlet of volute duct.

\section{Optimization algorithm and description of blade parameterization}

IOSO software was used as optimization program [5]. This program has proven itself in many research studies [11-13]. The optimization algorithm for the investigated pump is shown in Figure 6, and its description is presented below.

At each step of optimization, the optimizer IOSO PM generates a Vector of variable parameters $x_{1}, x_{2}, x_{3}, \ldots, x_{n}$. The Vector describes the geometry of the LPI, TP and HPI blades in a parametric form. The Vector of variable parameters is transferred to the reprofiling block, at which Numeca AutoBlade [9] program perform a conversion of the blades by Vector data and save them in the form of geometry files in a *.GeomTurbo format. Then, the mesh model is created in the Numeca AutoBlade 5 program using new blades. At the next step, the CFD calculation is performed with the new mesh model. The processing of CFD-results is carried out by a special script, using ANSYS CFX [10] small applications and NET Framework library. As a result, several output files are created containing operation parameters of the pump in the text format. These parameters are then passed to the optimizer IOSO.

The optimization goal was the increase of the pump efficiency with preservation or slight increase in the pressure head. This will reduce the load of the main gas turbine. It is necessary because the main gas turbine of the turbopump unit operates in oxidizing environment (the mass ratio at the gas generator is more than 56 for oxygen/kerosene components).

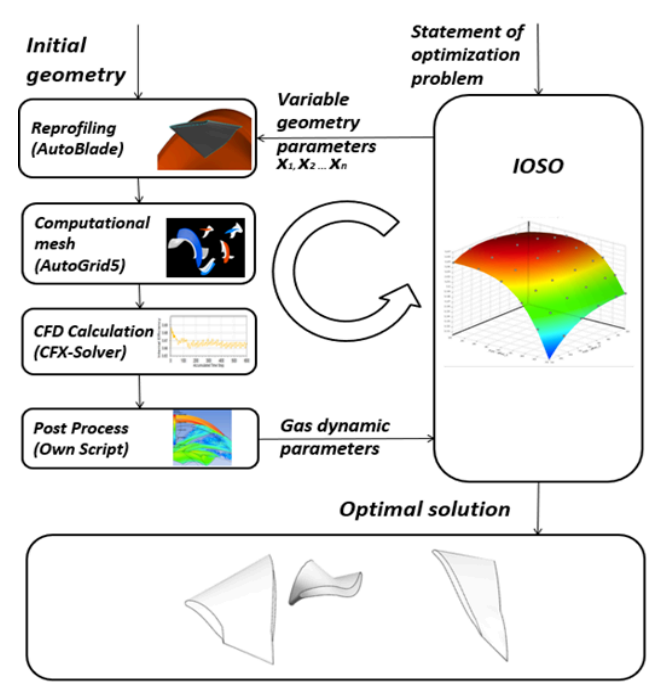

Figure 6. The optimization algorithm of the investigated pump.

In this study, the parameters of camber lines of the LPI, TP and HPI blades for two sections (hub and shroud) were chosen as optimization parameters. Figure 7 shows the parameterization scheme of the LPI blades. The camber lines of the LPI, TP and HPI blades are described by Bezier curves. The camber line of the LPI blade is described by three points-poles, while the camber lines of the TP and HPI requires at least four point-poles. This is due to the large length of the TP and HPI blades. The distribution of the cross sections along the blade height was carried out according to the linear law.

The fitting of the blades was performed in the Numeca AutoBlade. The blades of LPI, TP and HPI were reprofiled by only 2 sections (hub and shroud). The camber lines of the blades were specified by parameters of Bezier curves with three or four point-poles. The total number of independent variables was 22 .

The pump model with fitted blades was called $v 0$, and a comparison of its characteristics with the basic values is shown in the Figures 8 and 9. Analysis of these data shows that the pressure head differs from the basic value by $20 \mathrm{~m}$, or $1 \%$, and the efficiency differs by 0.013 or $2 \%$. A good match of the mesh $\mathrm{B} 2 \mathrm{Bm} 2$ level with a mesh $\mathrm{B} 2 \mathrm{~B} 0$ level is revealed.

Modifying only the camber lines assumes that the stresses in the blades will remain approximately the same as the original values. For the optimal version, a strength analysis was carried out.

In this research, two optimization studies were carried out. The first one uses the pump CFD-model without KID and VOD parts. The second one uses the CFD-model with all pump parts. 


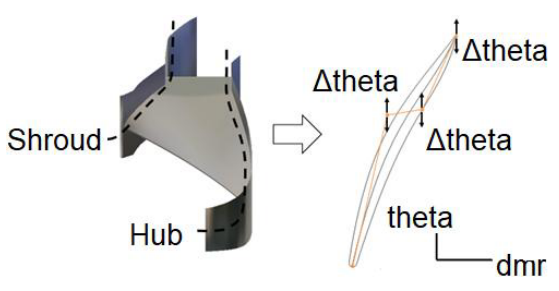

Figure 7. 2D parameterization of the LPI blades.

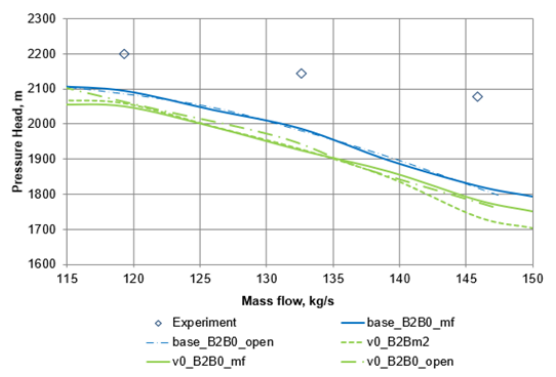

Figure 8. The pressure head characteristic of the pump for basis and $\mathrm{v} 0$ versions of LPI, TP and HPI blades.

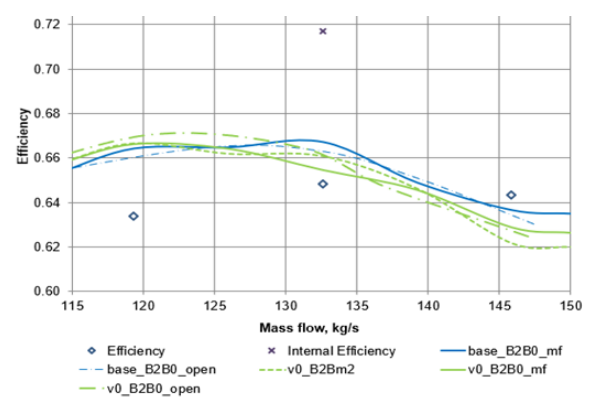

Figure 9. The internal efficiency characteristic of the pump for base and v0 versions of LPI, TP and HPI blades.

\section{Simplification of the pump model for optimization}

Simplification of the pump model is necessary to reduce the estimated time of CFD-calculation.

Simplified models were calculated with the Mass Flow boundary condition, since changing the geometry of the LPI, TP, and especially of the HPI can lead to a displacement of the pump characteristics.

Further, verification calculations were carried out with mesh model settings from the basic version of the pump and with two boundary conditions: Mass Flow and Opening Pressure for pump variants with the optimal blade geometry.

In this paper, the simplification of the pump model included the following studies:

- - comparison of the results of simulations with cavitation and without one;

- - comparison of the results of stationary and transient (without cavitation) simulations of pump workflows;

- - study of the possibility of optimization with a CFD model that does not include KID and VOD units;

- - a study of the pump characteristics behavior, depending on the mesh model level in the blades passages (B2B section) and on the flowpath amounts.

\subsection{Comparison of the results of simulations with cavitation and without one}

The phenomenon of cavitation should be considered in hydrodynamic investigations of the pumps, because the water hammer effect, arising from the cavitation, poses a serious risk to the pump reliability. Also, neglecting the cavitation simulation in pumps can lead to obtaining characteristics with a sufficiently large error of $10-15 \%$ $[14,15]$. To estimate this error for the pump, the modeling of working processes with cavitation was carried out. The simulation was performed with the basic mesh model. Cavitation settings were the following:

- doubled fluids: primary - liquid, secondary vapor;

- homogeneous model of multiphase under the assumption of the identity of the velocity field of vapor phase and liquid;

- cavitation model of Rayleight-Plesset for mass transfer;

- bubbles mean diameter is 2 microns;

- $\quad$ saturation pressure is $3169 \mathrm{~Pa}$;

- internal efficiency;

- volume fraction at inlet: " 1 " for liquid, " 0 " for vapor.

The results of comparison of the pump integral characteristics are shown in Figures 10 and 11. The pressure characteristics are practically the same, and the efficiency with cavitation is lower by $1.0-2.9 \%$. The exception is the area of high mass flow rate, where the characteristics of the pump, considering cavitation, become flat.

Simulation of the pump considering cavitation took in 1.9 times more resources than simulations without cavitation. The used processor-hours value is meant here under resources. At the same time, the pressure and efficiency characteristics are equidistant in the nearnominal mode. Therefore, the modeling of working processes in the pump was carried out without considering cavitation for further research.

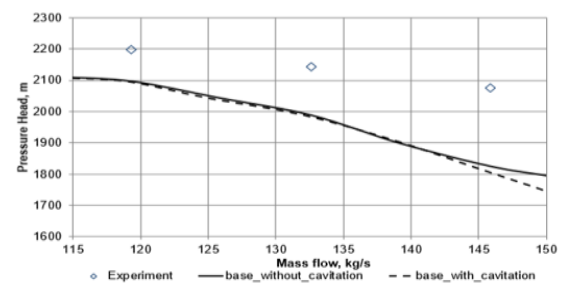

Figure 10. The pressure head characteristic of the investigated pump without and with cavitation modeling.

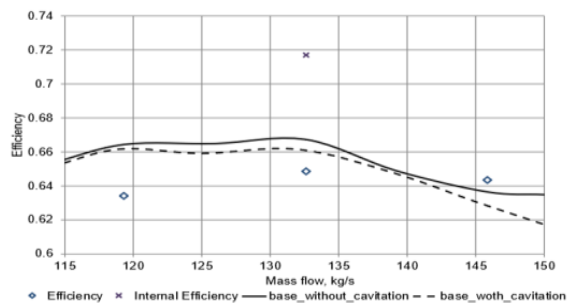

Figure 11. The internal efficiency characteristic of the investigated pump without and with cavitation modeling. 


\subsection{Comparison of the results of stationary and transient simulations of pump workflows}

In the "Methodology» section, the specific decision on stationary modeling with averaging the last 100 iterations was mentioned. This section contains the rationale for such approach.

The simulation was performed with the basic mesh model. Settings for the transient modeling were the following:

- the time step is $3.7594 \mathrm{e}-005$ seconds. This value corresponds to $1 / 10$ of the transit time of the HPI blade channel;

- Maximum Number of Coefficient Loops is 10 ;

- Number of Timesteps is 600.

The sustained operation of the pump required 300 iterations. The last 300 iterations (of total 600) were used for averaging the results of nonstationary calculation. The results of the comparison of the pump integral characteristics for steady and transient cases are shown in Figures 12 and 13.

Figures 12 and 13 show the integral characteristics of the pump for stationary and transient simulations that differ by less than $0.3 \%$, except for the low mass flow rate region for the efficiency characteristic.

The simulation of non-stationary pump operation was very resource-intensive task. So, even using only the first 300 iterations of transient simulation, the resourceintensity was 2.7 times more than for the stationary simulation (without cavitation). Regarding the foregoing, the simulation of working processes in the pump was carried out in the steady state (without cavitation) for further investigations.

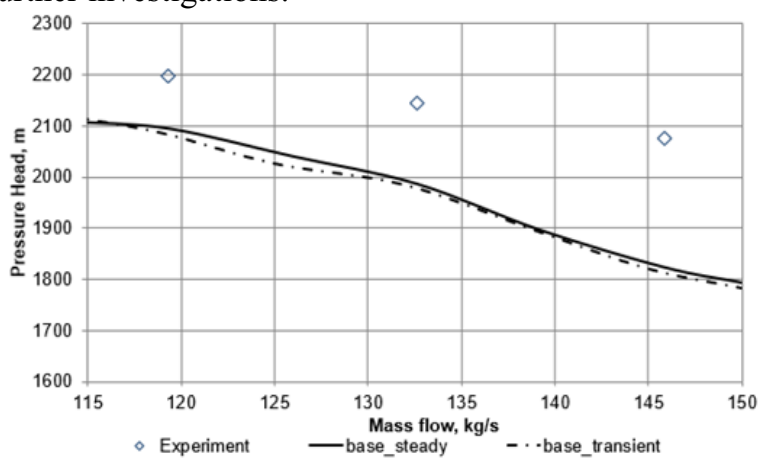

Figure 12. The pressure head characteristic of the investigated pump for steady and transient cases.

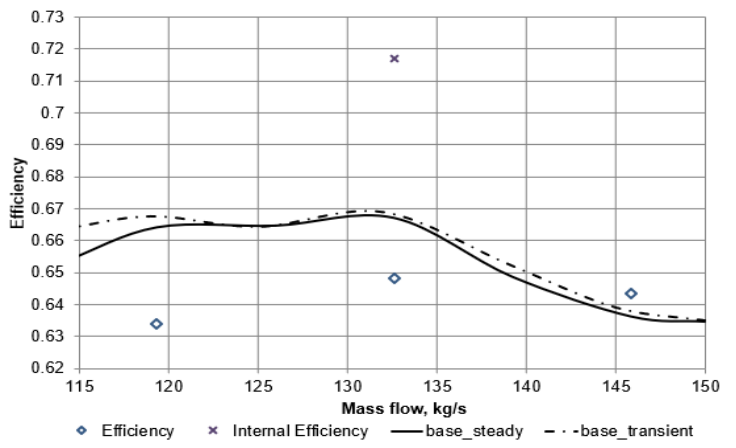

Figure 13. The internal efficiency characteristic of the investigated pump for steady and transient cases.

\subsection{Investigation of the optimization possibilities by using a CFD model without kid and vod parts of the pump}

The first optimization study by using CFD-model without KID and VOD parts provided an increased efficiency by $4 \%$ with increased pressure head by $6 \%$ at $\mathrm{B} 2 \mathrm{Bm} 2$ and B2B0 mesh levels (without KID and VOD parts). Optimization required only 100 iterations with the CFDmodel. Checking the optimal model with KID and VOD pump parts revealed a slight increase in efficiency by $0.2 \%$ with decrease in pressure head by $1.5 \%$. This was because the first CFD-model does not consider the influence of the VOD on the flow structure after the impeller.

Nevertheless, optimization with the first CFD-model correctly predicted trends in the geometry variation of the LPI and TP blades (same as with the second CFD-model). Thus, it is possible to perform pump blades optimization without VOD part, while maintaining the blades geometry in the stage before the outlet device.

\subsection{Investigation of the pump characteristics dependence on the mesh model}

Mesh generation for the vane units (rotors and stator transferring passage) was performed using NUMECA AutoGrid5 [9], which allows the creation of structural hexagonal mesh models.

The basic mesh model of the vane units contained 2.81 million elements $(3.08 \mathrm{mln}$ points $)$ with 81 flowpaths along the height.

The number of elements in the $\mathrm{B} 2 \mathrm{~B}$ section of the vanes of the basic model was adopted as B2B0 level. Two levels of B2B meshes were created with a reduced number of points: $\mathrm{B} 2 \mathrm{Bm} 1$ and $\mathrm{B} 2 \mathrm{Bm} 2$. The $\mathrm{B} 2 \mathrm{~B}$ levels differed by $\sim 1.3$ times according to the recommendations given in [9-11]. The number of points in 3D meshes depending on B2B level and flowpaths number, which are presented in Table 1. Flowpaths number is the number of elements along the height.

Table 1. The Number of Points in the 3D Mesh, $x 10^{6}$

\begin{tabular}{|c|c|c|c|c|}
\hline \multirow{2}{*}{$\begin{array}{c}\text { B2B } \\
\text { level }\end{array}$} & \multicolumn{4}{|c|}{ Flowpaths number } \\
\cline { 2 - 5 } & 81 & 73 & 65 & 57 \\
\hline B2B0 & 3.08 & 2.80 & 2.51 & 2.22 \\
\hline B2Bm1 & 2.28 & 2.10 & 1.85 & 1.64 \\
\hline B2Bm2 & 1.77 & 1.60 & 1.44 & 1.27 \\
\hline
\end{tabular}

The study showed that all mesh models adequately predict the values and behavior of the pressure head and internal efficiency characteristics. Figures 14 and 15 show the pressure head and efficiency characteristics of the investigated pump for several mesh models. The internal efficiency and pressure head values were determined at the outlet of HPI stage, to determine the influence of only the mesh parameters of the blade elements on the pump characteristics.

The maximum difference of the pressure head at the nominal mode for B2Bm2 flowpath57 mesh model was $1 \%$ relative to the basic value, and of the internal 
efficiency - $0.2 \%$ for the for B2B0_flowpath57 mesh model. The mesh model B2Bm2 flowpath57 was chosen for the optimization task, because it provides the fastest possible solution time from the considered mesh models, while at the same time it provides an adequate prediction of the pump characteristics.

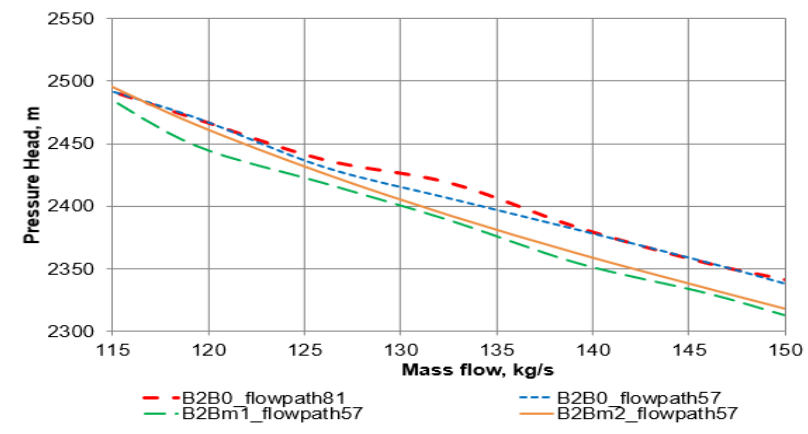

Figure 14. The pressure head characteristic of the investigated pump for several mesh models determined at the outlet of the HPI stage.

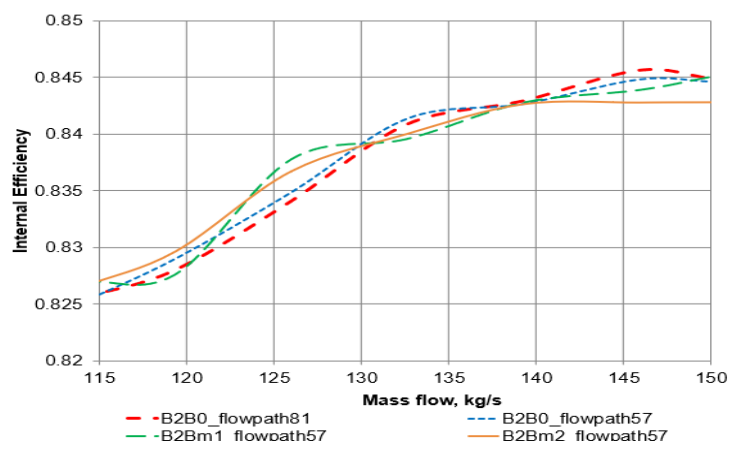

Figure 15. The internal efficiency characteristic of the investigated pump for several mesh models determined at the outlet of the HPI stage.

\section{Optimization results by using CFD- model with all parts of the pump}

The second CFD-model contains the mesh model v0 of blades and mesh models of KID and VOD from rough mesh of previous study [3].

Gas-dynamic optimization of the working process of the powerful fuel pump was performed. The gas-dynamic optimization task of the pump had two criteria:

- increase in the internal efficiency;

- preservation or a slight increase of the pressure head.

The gas-dynamic optimization task was performed using mesh model of B2Bm2 level.

The gas-dynamic optimization of the pump required more than 200 iterations with second CFD-model. As a result of the optimization, the Pareto front between the efficiency and pressure head values was obtained (Fig. 16).

For a detailed analysis, 2 points were selected from the Pareto front of pump optimization (indicated by the arrows in Fig. 16):
- point v33 is the variant of the pump with increased internal efficiency by $3.3 \%$ and increased pressure head by $6.8 \%$ at $\mathrm{B} 2 \mathrm{Bm} 2$ mesh level relative to variant $v 0$;

- point $\mathrm{v} 41$ is the variant of the pump with increased internal efficiency by $5.4 \%$ and increased pressure head by $0.2 \%$ at $\mathrm{B} 2 \mathrm{Bm} 2$ mesh level relative to variant $v 0$.

Then calculations of variants v33 and v 44 were performed at B2B0 mesh level. The internal efficiency and pressure head characteristics with boundary condition Mass Flow at the outlet are shown in Figures 17-18, and with boundary condition Opening are in the Figures 1920. The Figures 17-20 show that pressure head of pump variant $\mathrm{v} 41$ is below the basic value at the parity level of the internal efficiency in comparison with pump variant v33. In addition, the internal efficiency of the pump is sharper in the field of high mass flows. The pump variant v33 seems preferable.

The internal efficiency of the pump variant v33 has the increased value by $3.1-4.5 \%$ and the increased pressure head by $0.4-3.9 \%$ at nominal mode relative to the basic values, depending on the boundary condition.

Figure 21 shows the blade-to-blade sections of LPI, TP and HPI pump blades of basic and optimized (v33) versions. LPI blade became more elongated closer to the exit, the blade of the TP became more curved, and provides a certain flow angle for the HPS. The HPI blade underwent major change closer to the outlet of HPI stage, especially at the shroud. It seems, that such shape of the HPI blade ensures compensation of Pressure Head losses in the periphery of the HPS blade.

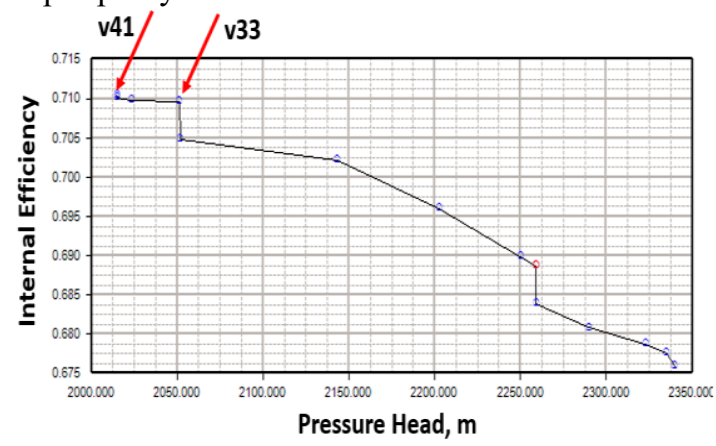

Figure 16. Pareto front «Internal Efficiency - Pressure Head» of the investigated pump.

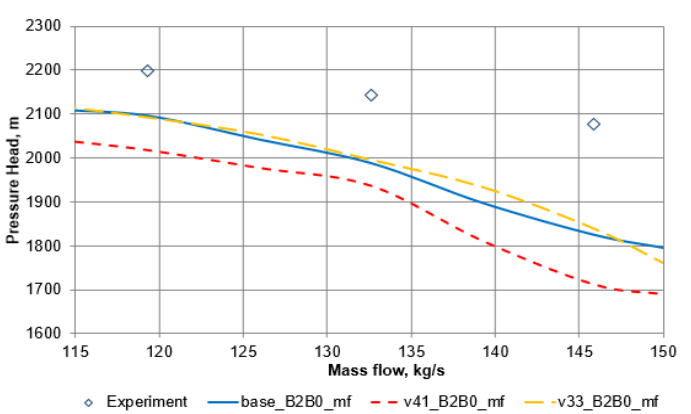

Figure 17. The pressure head characteristic of the pump with boundary condition Mass Flow. 


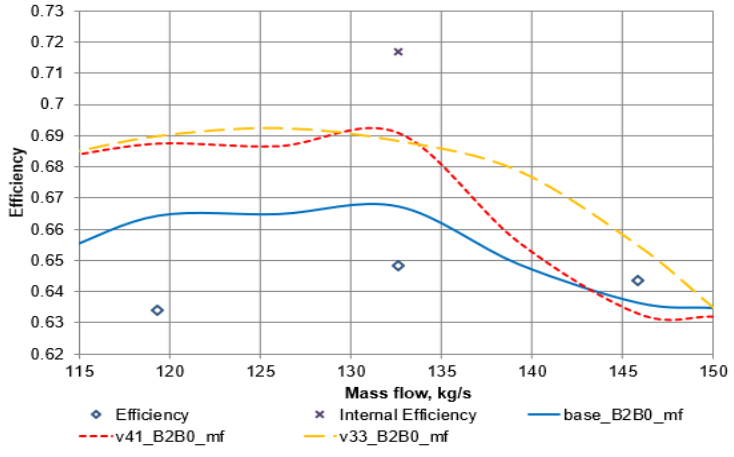

Figure 18. The internal efficiency characteristic of the pump with boundary condition Mass Flow.

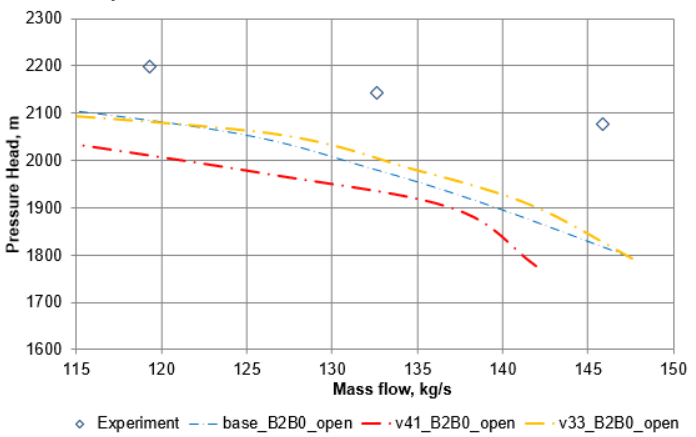

Figure 19. The pressure head characteristic of the pump with boundary condition Opening.

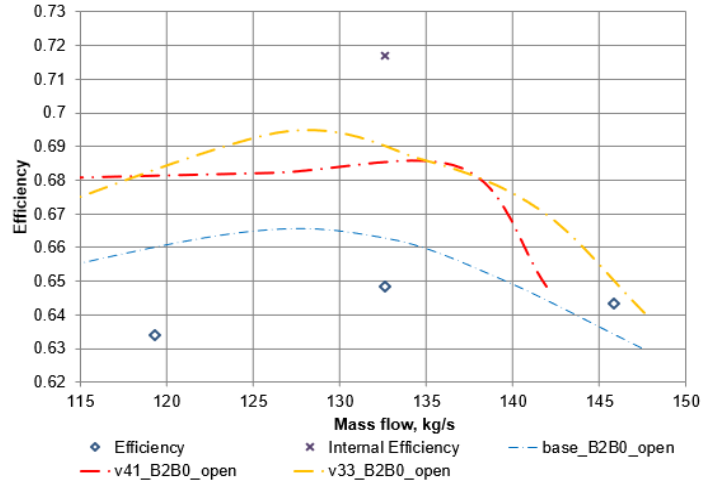

Figure 20. The internal efficiency characteristic of the pump with boundary condition Opening.

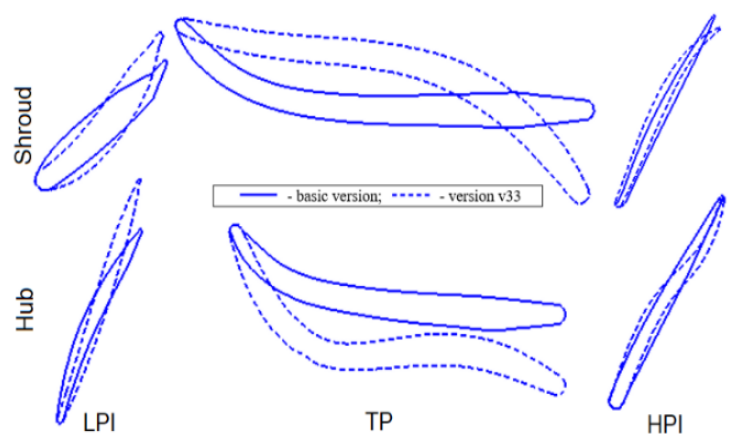

Figure 21. Blade-to-blade sections of LPI, TP and HPI pump blades of base and optimized (v33) versions.

Analysis of the pressure head and efficiency parameters by the pump parts was carried out for the basic pump, v33 and v44 variants. Changing the parameters of internal efficiency and pressure recovery coefficient is shown in Figure 22, from which it can be seen that the greatest change occurred in the transferring passage (TP) of the pump.

Fig. 22 shows that the pump variant v44 has higher efficiency for the impeller than the pump variant $v 33$, but $\mathrm{v} 44$ has lower pressure recovery coefficient for the VOD. Apparently, the calculation on the fine B2B0 mesh level allowed to calculate the vortices shown in Figures 3 and 5 more detailed.

Since only the LPI, TP and HPI blades were subject to optimization, the efficiency of the LPS and HPS blades should remain at the same level or slightly change. The efficiency of the LPS blades slightly increased due to the change in the shape of the LPI blades. At the same time the efficiency of the HPS blades decreased because of the change in the velocity triangles associated with the change in the shape of the TP and HPI blades. In general, the efficiency of the pump increased by $3.1-4.5 \%$ with the increased pressure head by $0.4-3.9 \%$ due to the coordinated work of the stages [16].

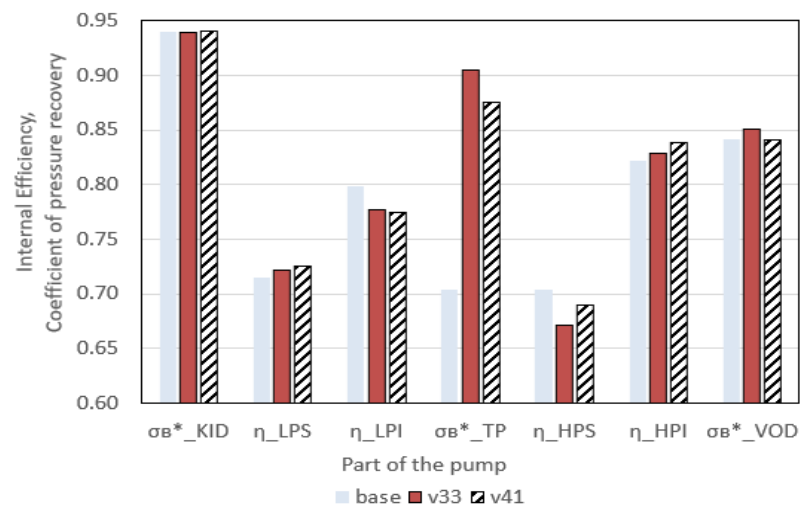

Figure 22. The parameters of internal efficiency and pressure recovery coefficient determined for the pump parts

\section{Strength analysis of HPI blade optimal geometry}

The geometry of the HPI blades was significantly changed during the optimization. To evaluate the effect of loads acting on the optimized variant of geometry, calculations are made to determine the maximum equivalent stresses in the basic geometry and in the optimized variant of the high-pressure impeller. The calculation was carried out in ANSYS Mechanical. Computational model was the impeller sector, the cyclic symmetry condition was used. The calculation did not consider the material ductility. The pressure values on the solid surfaces were interpolated from the CFD calculation. The temperature field was $25^{\circ} \mathrm{C}$, given as constant for the entire calculation model.

As a result of the calculation of two impeller variants, the values of the maximum equivalent stresses were obtained. For the basic variant the maximum value of equivalent stress $\sigma \_(\text {von Mises stress)_base was } 961.6$ $\mathrm{MPa}$, for the optimized variant v33 - $\sigma_{-}($von Mises stress)_opt was 1038.3 MPa.

The material of HPI is chromium-nickel steel VNL-1. It has the specific stress limit of $1079 \mathrm{MPa}$ [17]. 
According to J. E. Shigley «stress-concentration factors need not be employed when the material is ductile, and the loads are static») [18].

Figures 23 show the distribution of the equivalent stress parameter in the HPI blade of basic and optimized v33 versions. According to the recommendations in [17] and [19] «failure is most often declared if yielding occurs across a complete section». Therefore, the region of the equivalent stresses near the maximum value was considered in this study. A region occupying 1/4 of the maximum stress scale for each case was adopted as such region. The equivalent stresses for the selected region was $722.3 \mathrm{MPa}$ for the basic HPI blade version and 779.2 $\mathrm{MPa}$ - for the optimized v33 version. Then the safety factor for the basic version is 1.49 , and for the optimized version is 1.38 .

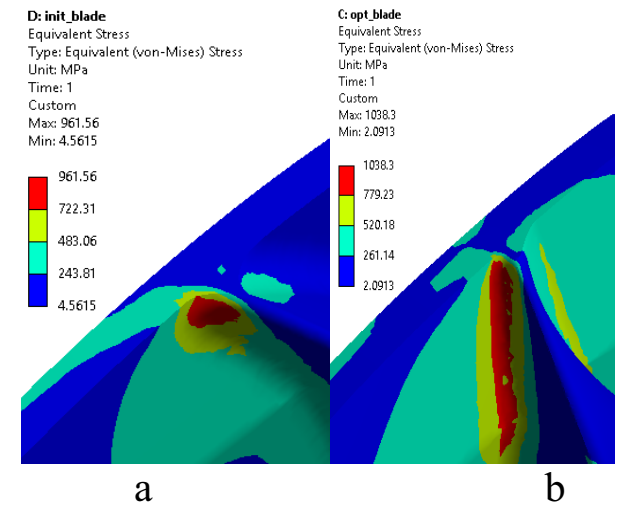

Figure 23. Distribution of the equivalent stress parameter in HPI blade of basic (a) and v33 (b) versions .

\section{Conclusions}

Two optimization studies of powerful fuel pump were performed. The first optimization task with CFD-model, which does not contain KID and VOD parts of the pump, did not allow to significantly increase the pressure head and internal efficiency. Nevertheless, optimization with the first CFD-model correctly predicted the trends in the geometry variation of the LPI and TP blades. Thus, it is possible to perform optimization of the pump blades without VOD part, while maintaining the geometry of the blades in the stage before the output device. This can be important for the gradual improvement of the pump geometry, especially if the number of variable parameters is limited by the capabilities of an optimizer program.

The second optimization task provided the pump with re-profiling geometry of the blades, which allow to obtain the increased internal efficiency by $3.1-4.5 \%$ and the increased pressure head by $0.4-3.9 \%$ at nominal mode relative to the basic values.

The strength analysis of the HPI blade was performed since the HPI blades were greatly changed at the HPI stage exit area. The maximum equivalent stresses increased by $76.7 \mathrm{MPa}$, and the safety factor of HPI decreased from 1.49 to 1.38 .

The obtained reserve can be used to boost the rocket engine, and/or to reduce the loading of the main turbine, which operates in aggressive oxidizing environment.
Further optimization is planned for 3-4 sections for all blade stages, including screws. Also, conjugate optimization is planned to consider the strength model.

\section{Acknowledgments}

This work was financially supported by the Ministry of education and science of the Russian Federation in the framework of the implementation of the Program "Research and development on priority directions of scientific-technological complex of Russia for 20142020 " with using equipment of CAM technology shared center retrofitted by project RFMEFI59314X0003.

\section{References}

1. Andronov, A.L., "Features of operation of centrifugal pumps and the requirements for their electric drive," Polzunovskii Almanac (Barnaul, Russia), Volume 1, pp. 150-152, (2004)

2. Ivanov, V.K., Kashkarov, A.M., Romacenko, E.N., Tolstikov L.A., "Turbopump units for LPRE designed by NPO Energomash," Conversion in engineering, Volume 1, pp. 15 21,(2006)

3. Zubanov, V.M., Shabliy, L.S., Krivcov, A.V.,"Rational Technique for Multistage Centrifugal Pump CFD-Modeling," In Proceedings of the ASME Turbo Expo, Paper No. GT2015-42070, pp. 19,(2015)

4. Zubanov, V.M., Shabliy, L.S., Krivcov, A.V., Matveev, V.N., " Document Technique for adequate CFD-modeling of the pump with hydro-drive of the low-pressure stage," In Proceedings of the ASME Turbo Expo, Paper No. GT2016-57689, pp. 111,(2016)

5. IOSO Optimization Technology. Access mode: http://www.iosotech.com

6. Pinho, J., Lema, M., Rambaud, P., Steelant, J., 2014, "Multiphase investigation of water hammer phenomenon using the full cavitation model," Journal of Propulsion and Power, Volume 30, Issue 1, January, pp. 105-113Fg, (2014)

7. Reboud JL., Pouffary B., Coutier-Delgosha O., Fortes - Patella R., "Numerical simulation of unsteady cavitating flows: some applications and open problems," Fifth International Symposium on Cavitation (CAV2003), Japan, Paper No. CAV2003IL-10, pp. 1-10,(2003)

8. NUMECA. Access mode: http://www.numeca.com/en

9. ANSYS CFX-Solver Modeling Guide, ANSYS Inc, ( 2011)

10. Marchukov, E.Yu., Egorov, I., Popov, G., Salnikov, A., Goriachkin, E., Kolmakova, D., " Multidisciplinary optimization of the working process of uncooled axial turbine according to efficiency and strength criteria," Proceedings of the ASME Turbo Expo, Paper No. GT2017 64843,(2017)

11. Jha, R., Dulikravich, G.S., Colaço, M.J., Egorov, I.N., Poloni, C., Chakraborti, N., Fan, M., Schwartz, J., Koch, C., 2015, " Multi-objective 
design and optimization of hard magnetic alloys free of rare earths," Materials Science and Technology Conference and Exhibition (2015).

12. Matveev, V.N., Baturin, O.V., Popov, G.M., Egorov, I.N., " Document Seven-stage axial compressor optimization," Engineering Optimization IV Proceedings of the 4th International Conference on Engineering Optimization, ENGOPT 2014, pp. 821826,( 2014)

13. Yang, C., Wu, H., "Optimized aerodynamic design of aggressive intermediate turbine duct with strut fairings using genetic algorithms", ASME Paper GT2016-56639,(2016)

14. Ding, H., Visser, F.C., Jiang, Y., Furmanczyk, M., "Demonstration and validation of a 3D CFD simulation tool predicting pump performance and cavitation for industrial applications," Journal of Fluids Engineering, Transactions of the ASME Volume 133, Issue 1, Article number 011101, pp. 277-293,( 2011)

15. Athavale, M.M., Li, H.Y., Jiang, Y.U., Singhal, A.K., "Application of the full cavitation model to pumps and inducers," International Journal of Rotating Machinery, Volume 8, Issue 1, pp. 45-56,( 2002)

16. Popov G., Goriachkin E., Kolmakova D. and Novikova Yu., "Multicriteria Optimization of Axial Low Pressure Compressor of Gas Turbine Power Plant",( 2016)

17. https://catalog.viam.ru/

18. Shigley J.E. and C.R., "Mechanical Enginering Design", McGraw-Hill, (2001).

19. ANSYS Mechanical User's Guide, ANSYS,( 2013) 